\title{
Cost-Effective EEG Signal Acquisition and Recording System
}

\author{
Sabbir Ibn Arman, Arif Ahmed, and Anas Syed
}

\begin{abstract}
This paper describes a viable, low cost Electroencephalogram (EEG) signal acquisition device which collects and records the signals in the desktop computer using DSP Processor DS-1104. The main considerations while making such a device are simplicity, ease of use and applicability. The uniqueness of this device is that it uses only three electrodes while most other systems use the 10-20 system of electrode placement which requires 21 electrodes. But the reduction in electrodes must not invalidate the output of the system, which is the EEG signal. For this reason, it is critical that the electrodes are placed in the scalp location where we can observe distinguishable change in the various wave patterns of the EEG signal. The main circuit is made of simple electronic elements. The circuit consists of an amplifier, filter and an opto-isolator. In this paper, EEG signal acquisition principles are discussed, applied, and some suggestions for improving signal acquisition are presented.
\end{abstract}

Index Terms-EEG, brain computer interface, amplifier, electrodes, DS-1104.

\section{INTRODUCTION}

The human brain is always active. The brain controls the different activities of the body. The brain functions can be monitored by observing the electrical signals generated in the neurons. This signal is called electroencephalogram (EEG) signal. The signal can be extracted using electrodes and can be viewed using a voltmeter, oscilloscope or on a computer screen. The EEG signal can be recorded and the phenomenon is known as electroencephalography [1]. The EEG signal can be used to investigate the condition of a human brain and the overall health of the person. These signals are roughly less than $100 \mu \mathrm{V}$ and $100 \mathrm{~Hz}$ and can be measured with electrodes placed on the scalp, noninvasively [2], [3]. Because of their low amplitude due to the skull's composition, the measurement of EEG is more difficult than the other non-invasive bio-signal measurements such as the electrocardiogram (ECG), electromyogram (EMG), electrooculogram (EOG) and so forth [2]. Having expensive bio-signal recording systems cannot guarantee acquiring proper signals.

\section{EXISTING METHODS FOR EEG ACQUISITION}

Review Electrodes are used to obtain the EEG signals from the scalp of the human brain. The EEG recording electrodes

Manuscript received August 1, 2012; revised September 10, 2012.

The authors are with the Department of Electrical and Electronic Engineering, Islamic University of Technology (IUT) Board Bazar, Gazipur 1704, Bangladesh (e-mail: sabbir.arman@gmail.com, arifahmedeee@yahoo.com, syeed.anas@gmail.com). and their proper function are crucial for acquiring high quality data.

Different types of electrodes are often used in the EEG recording systems, such as:

- Disposable electrodes (gel-less, and pre-gelled types);

- Reusable disc electrodes (gold, silver, stainless steel, or tin);

- Headbands and electrode caps;

- Saline-based electrodes;

- Needle electrodes [2]

For multi-channel recordings with a large number of electrodes, electrode caps are often used. Commonly used scalp electrodes consist of $\mathrm{Ag}-\mathrm{AgCl}$ disks, less than $3 \mathrm{~mm}$ in diameter, with long flexible leads that can be plugged into an amplifier [2]. Silver chloride $(\mathrm{AgCl})$ is preferred for common neurophysiologic applications. Because $\mathrm{Ag}$ is a slightly soluble salt, $\mathrm{AgCl}$ quickly saturates and comes to equilibrium. Therefore, $\mathrm{Ag}$ is a good metal for metallic skin-surface electrodes [4], [5]. The International Federation of Societies for Electroencephalography and Clinical Neurophysiology has recommended the conventional electrode setting (also called 10-20) for 21 electrodes (excluding the earlobe electrodes), as depicted in Fig. 1. The "10" and "20" refer to the fact that the actual distances between adjacent electrodes are either $10 \%$ or $20 \%$ of the total front-back or right-left distance of the skull [2], [6].

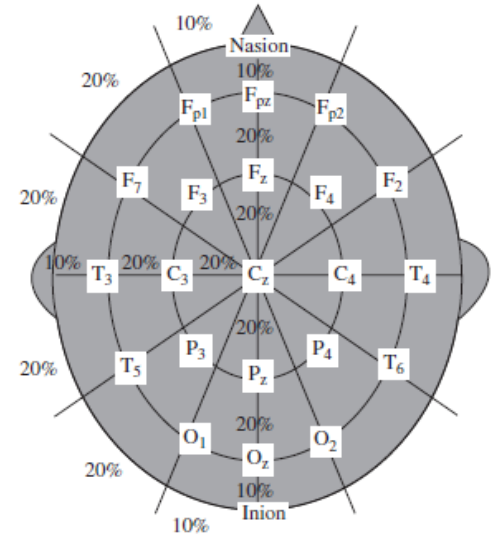

Fig. 1. Conventional 10 - 20 EEG electrode position for the placement of 21 electrodes.

For setting a larger number of electrodes using the above conventional system, the rest of the electrodes are placed in between the above electrodes with equidistance between them. For example, $C_{1}$ is placed between $C_{3}$ and $C_{z}$. Fig. 2 represents a larger setting for 75 electrodes including the reference electrodes based on the guidelines by the American

EEG Society. Extra electrodes are sometimes used for the measurement of EOG, ECG, and EMG of the eyelid and eye surrounding muscles. In some applications such as ERP 
(Event-Related Potentials) analysis and brain computer interfacing a single channel may be used. In such applications, however, the position of the corresponding electrode has to be well determined. For example, $C_{3}$ and $C_{4}$ can be used to record the right and left finger movement related signals respectively for brain-computer interfacing (BCI) applications. Also F3, F4, $\mathrm{P}_{3}$ and $\mathrm{P}_{4}$ can be used for recording the ERP P300 signals [7].

In another similar setting, called the Maudsley electrode positioning system, the conventional 10-20 system has been modified to capture better the signals from epileptic foci in epileptic seizure recordings. The only difference between this system and the 10-20 conventional system is that the outer electrodes are slightly lowered to enable better capturing of the required signals. The advantage of this system over the conventional one is that it provides a more extensive coverage of the lower part of the cerebral convexity [8],[9],[10].

Since EEG signal represents voltage difference between two electrodes, one or more electrodes have to be set as reference so that the output voltage can be measured with respect to that reference point. There are various systems of taking reference [6], most common being the linked ears, which is an average of the voltage of the electrodes attached to either earlobes or mastoids.

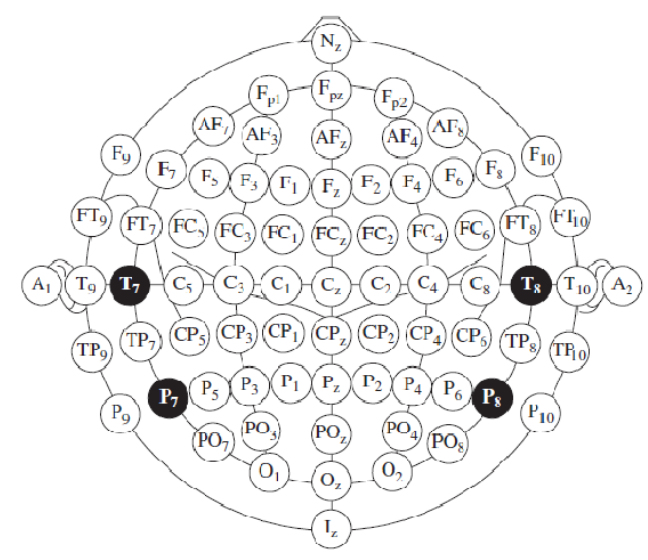

Fig. 2. A diagrammatic representation of 10-20 electrode settings for 75 electrodes including the reference electrodes: (a) and (b) represent the three-dimensional measures, and (c) indicates a two-dimensional view of the electrode setup configuration.

The raw EEG signals obtained from the electrodes have amplitudes of the order of micro volts and contain frequency components of up to $300 \mathrm{~Hz}$. The signal is amplified approximately ten thousand times [2].

High pass filters with a cut-off frequency of usually less than $0.5 \mathrm{~Hz}$ are used to remove the disturbing very low frequency components such as those of breathing. On the other hand, high-frequency noise is mitigated by using low pass filters with a cut-off frequency of approximately 50-70 $\mathrm{Hz}$. Notch filters with a null frequency of $50 \mathrm{~Hz}$ are often necessary to ensure perfect rejection of the strong $50 \mathrm{~Hz}$ power supply [2]. The signals are converted to digital form by using ADC to store the signal in a computerized system.

\section{Proposed Method of EEG Signal ACQuisition}

The acquired EEG signal is amplified using precision instrumentation amplifier AD624. An outstanding combination of low noise, high gain accuracy, low gain temperature coefficient and high linearity make the AD624 ideal for use in high resolution data acquisition systems. The AD624 provides totally independent input and output offset nulling terminals for high precision applications. This minimizes the effect of offset voltage in gain ranging applications. The AD624 is a functionally complete instrumentation amplifier. Pin programmable gains of 1, 100, 200, 500 and 1000 are provided on the chip. Other gains are achieved through the use of a single external resistor. A sense terminal is provided to enable the user to minimize the errors induced through long leads. A reference terminal is also provided to permit level shifting at the output [11]. The output of the AD624 is then passed through a low pass Butterworth filter. The output of the filter is then passed through an opto-isolator.

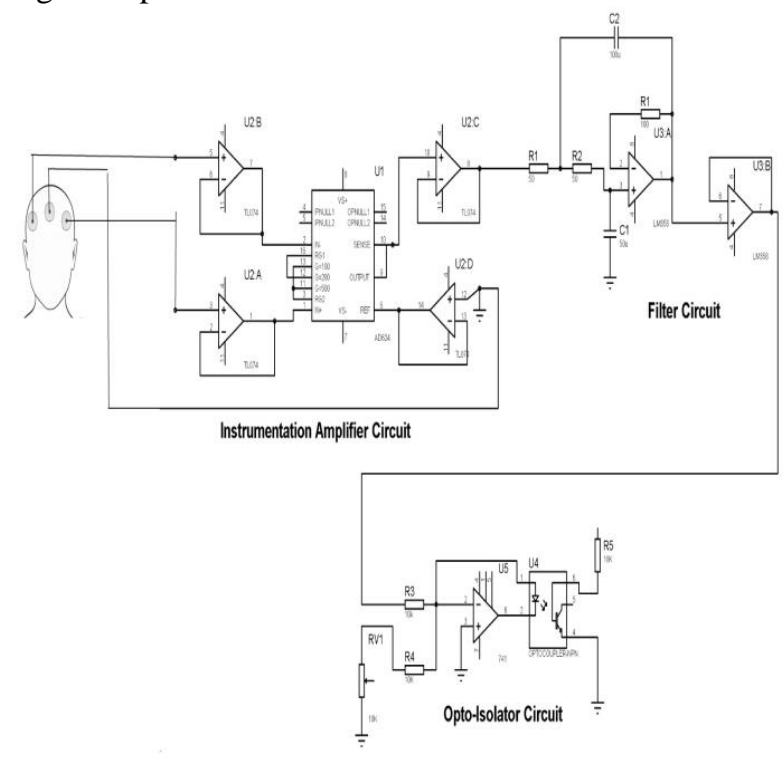

Fig. 3. EEG Acquisition circuit schematic diagram.

The conventional electrode setting (10-20) has not been used for this system. Three electrodes are sufficient to acquire EEG signal compared to 10-20 system. It is less complicated in terms of analysis and data processing. In our system, we use disposable metal buckle electrodes. One electrode is placed at the centre of the forehead. This electrode is used as reference. Two electrodes are placed at the left and right of the forehead. The left electrode is connected to the inverting terminal (pin 1) of the AD624 and the right electrode is connected to the non-inverting terminal (pin 2) of the AD624. The electrodes are connected to the AD624 via buffer amplifiers or voltage followers.

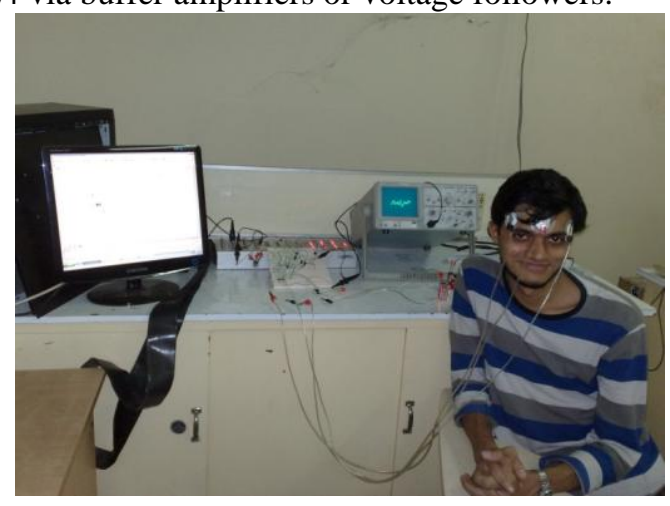

Fig. 4. EEG Signal acquisition and recording system. 


\section{BRAIN COMPUTER INTERFACE (BCI)}

A Brain-Computer Interface (BCI) is a system that acquires and analyzes neural signals with the goal of creating a communication channel directly between the brain and the computer. Such a channel potentially has multiple uses.

- Bioengineering applications: Assist devices for disabled people.

- Human subject monitoring: Sleep disorders, neurological diseases, attention monitoring, and/or overall "mental state".

- Neuroscience research: Real-time methods for correlating observable behavior with recorded signals.

- Man-Machine Interaction: Interface devices between human and computers, machines, etc [12].

The common structure of a Brain Computer Interface is the following:

- Signal acquisition: the eeg signals are obtained from the brain through invasive or non-invasive methods (for example, electrodes). After, the signal is amplified and sampled.

- Signal pre-processing: once the signals are acquired, it is necessary to clean them.

- Signal classification: once the signals are cleaned, they will be processed and classified to find out which kind of mental task the subject is performing.

- Computer interaction: once the signals are classified, they will be used by an appropriate algorithm for the development of a certain application [12].

Then the amplified output is passed through a low pass Butterworth filter with cut off frequency $45 \mathrm{~Hz}$. The EEG signals contain neuronal information below $100 \mathrm{~Hz}$ (in many applications the information lies below $30 \mathrm{~Hz}$ ). The important EEG wave groups (alpha, beta, theta, gamma, delta and $\mathrm{mu}$ ) fall within $45 \mathrm{~Hz}$ range. Any frequency component above these frequencies can be simply removed without any loss of information [12]. The filtered signal is then passed through 4N35 opto-isolator. The opto-isolator is used to totally isolate circuitry from mains electricity. An opto-isolator (or optical isolator, optical coupling device, optocoupler, photocoupler, or photoMOS) is a device that uses a short optical transmission path to transfer an electronic signal between elements of a circuit, typically a transmitter and a receiver, while keeping them electrically isolated - since the electrical signal is converted to a light beam, transferred, then converted back to an electrical signal, there is no need for electrical connection between the source and destination circuits [13]. A DSP processor named ds-1104 is used to record the EEG signal in a computer.

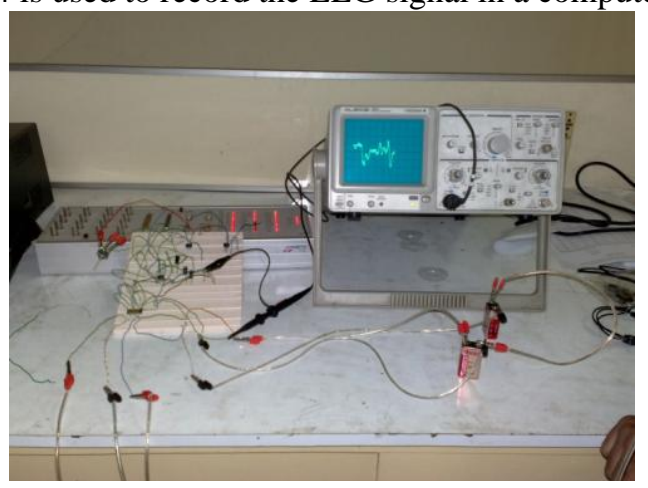

Fig. 5. EEG Signal acquisition and recording system (magnified).

\section{DS-1104 R\&D CONTROLLER BOARD}

The DS1104 R\&D Controller Board is a new piece of hardware that upgrades a PC to a powerful development system for rapid control prototyping. The real-time hardware based on Power PC technology and its set of I/O interfaces makes the board an ideal solution for developing controllers in various industrial fields. The new DS1104 R\&D Controller Board is impressive proof that power does not necessarily have to be expensive. The DS1104 offers an intelligent subset of the hardware architecture of the DS1103 PPC Controller Board. This way, the DS1104 is available at a reasonable price, making it the perfect development system for industry and equally for universities. Yet it still gives us all the benefits of a dSPACE Prototyper system: full graphical configuration, programming in Simulink/Stateflow from The MathWorks and experiment control with state-of-the-art software tools. The board can be installed in virtually any PC with a free PCI slot. The characteristics of DS1104 R\&D controller board are:

- Cost-effective system for controller development.

- Single-board PCI hardware for use in PCs.

- Set of intelligent I/O on-board.

- Fully programmable from the Simulink block diagram environment [14].

Through the help of DS-1104 EEG signal is stored in computer file which is shown in Fig. 6.

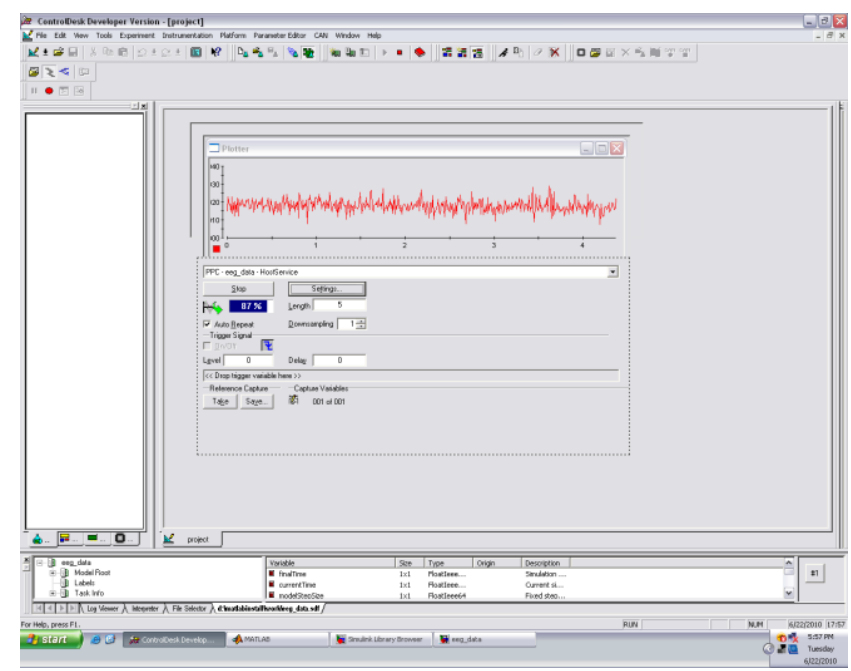

Fig. 6. EEG Signal in DS-1104 control centre.

\section{FEATURES}

- The system is portable - The main circuit is arranged within a bread board.

- Installation is easier than other device - Only three electrodes are connected to the test subject.

- The system is cost effective - The main circuit consists of electronic elements which are low-priced.

- Simple circuit - The circuit consist of an amplifier, filter and isolator.

- Filtering, sampling, digitizing is done using software.

- Real Time analysis - Data can be observed and recorded using DS-1104.

- Easy artifact removal - The signal can be manipulated using software and any unwanted frequency can be removed. 


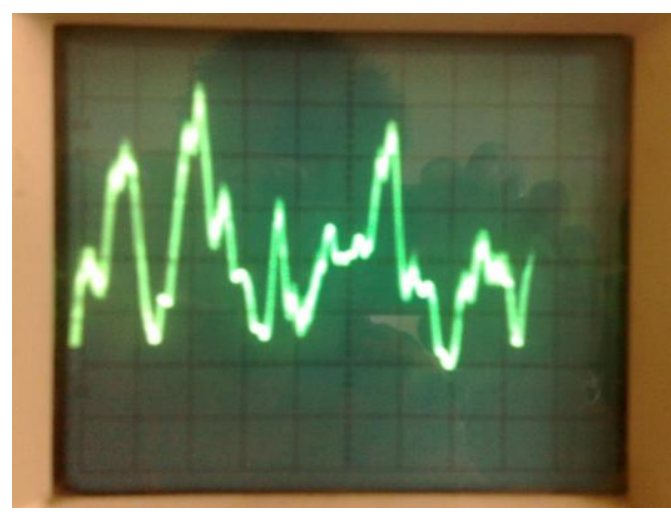

Fig. 7. EEG Signal in oscilloscope

\section{PROBLEMS FACED IN HARDWARE IMPLEMENTATION}

- Unavailability of high quality electrodes.

- Making the system noise free.

- Proper Electrode placement and Electrode life.

- Developing effective real time signal for collecting and analyzing the signals filtering the EMG signal from EEG signal.

- Stabilized Power Supply - Adapter.

- For reliable results repeated checking of connections was performed due to undependable breadboard connections.

\section{FURTHER DEVELOPMENT}

There are major points that should be considered to improve the measurement performance in the design of the bio-signal measurement system or recording session. Specifically choosing the correct electrode, skin preparation, and reduction of power line noise are the important issues for EEG recordings. To reduce electromagnetic interferences, a metal box for electronic circuits, a shielded (Faraday cage principle) recording room, and guarding (driven or not) for common mode signal reduction are the efficient methods. The performance of the bio-signal measurement system depends on the electrodes, electronic circuitry, and recording conditions. Choosing the correct electrode and successful electronic design strategy are essential to acquire EEG signals properly.

\section{CONCLUSION}

The device is single channel which is both an advantage and a disadvantage because it is simple but it also limits the scope of analysis. The device can be used just as effectively to observe and record ECG or EMG. Better result can be expected if the quality of the electrodes is improved. A notch filter can be used to eliminate the $50 \mathrm{~Hz}$ power supply noise.
The device is used to acquire data for different activities with different subjects. The data is stored in a computer file using the DS-1104 processor. The data is then analysed using Matlab through performing Fourier transform by using the software EEGLAB downloaded from the internet. After analysis, we observe that the results obtained match more or less with the expected outcome. This justifies the use of this low cost EEG acquisition device for professional purposes. Our future plan is to use this device to measure of depth of anaesthesia and to analyse EEG signal for epileptic patient.

\section{ACKNOWLEDGMENT}

We would like to convey our heartiest thanks to Prof. Dr. Md. Ashraful Hoque, Mr. Shahedul Amin, Mr. Tahmid Latif (Department of Electrical and Electronic Engineering, Islamic University of Technology) and Prof. Dr. K.S. Rabbani (Department of Applied Physics, University of Dhaka) for their unbounded support to make this work a success.

\section{REFERENCES}

[1] The Spline-Laplacian in Clinical Neurophysiology: A Method to Improve EEG Spatial Resolution. Nunez, P L and Pilgreen, K L. 1991, J Clin Neurophysiol, pp. 397-413.

[2] S. Saeid and C. Jonathon. EEG Signal Processing. s.1.: John Wiley and Sons, 2007.

[3] B. A. Khalil and K. E. Musilus, Atlas of EEG and Seizure Semiology. s.1. : Elsevier, 2006.

[4] B. S. Andrew, S. Christopher, and G. Mason, "Basic electronics in clinical neurophysiology. [book auth.] Andrew S. Blum and Seward B. Rutkove," The Clinical Neurophysiology Primer. s.1.: Springer-Verlag New York, LLC, 2007

[5] P. David and N. Michael. Design and Development of Medical Electronic Instrumentation. Hoboken, NJ : John Wiley and Sons, Inc., 2005. 0-471-67623-3.

[6] The Spatial Location of EEG Electrodes: Locating the Best-Fitting Sphere Relative to Cortical Anatomy. VL, Towle, et al. 1993, Electroencephalogr Clin Neurophysiol., pp. 1-6.

[7] Robust EEG Channel Selection across Subjects for Brain-Computer Interfaces. Schröder, Michael, et al., EURASIP Journal on Applied Signal Processing, pp. 3103-3112, 2005.

[8] The Direct Control of Neural Processes by Reward and Punishment. Black, A. H. 2, American Scientist, vol. 59, pp. 236-245,1971.

[9] Walking from Thought. G, Pfurtscheller, et al. 2006, Brain Res., pp. 145-152.

[10] User Adaptive BCIs: SSVEP and P300 Based Interfaces. Beverina, Fabrizio, et al., PsychNology Journal, pp. 331-354, 2003.

[11] AD624 Datasheet - Precision Instrumentation Amplifier. DigChip IC Database. [Online]. Available: http://www.digchip.com/datasheets/parts/datasheet/041/AD624-pdf.p hp.

[12] O. J. Baztarrica, EEG Signal Classification for Brain Computer Interface Applications. s.1.: Ecole polytechnique federale De lausanne, 2002.

[13] Optocoupler - The Full Wiki. The Full Wiki. [Online]. Available: http://www.thefullwiki.org/Optocoupler.

[14] dSPACE - DS1104 R\&D Controller Board. dSPACE. [Online]. Available:

http://www.dspaceinc.com/en/inc/home/medien/papers/download_pag e.cfm?FileID=778\&view $=$ pdf 